\section{Auf regelmäßiges Back-up achten}

Die Cyberattacke „WannaCry", die gerade auch britische Kliniken und Praxen getroffen hat, macht erneut deutlich, wie wichtig regelmäßige Datensicherung ist. In großen Praxen sollte täglich, in kleineren ein wöchentliches Back-up auf einen externen Datenträger (z. B. Festplatte) erfolgen. Cave: Datenträger direkt nach dem Sichern vom Praxissystem trennen! Sonst könnte er bei einem Hackerangriff ebenfalls mit einem Trojaner oder Virus infiziert werden.

RebekkaHöhl

\section{Verschlüsselt kommunizieren}

Gerade in Kliniken und großen Praxen können Chats via "Mobile Devices" (Smartphone, Tablet-PC) den Infofluss beschleunigen. Die mobile Kommunikation, insbesondere von privaten Geräten, die auch dienstlich genutzt werden, birgt aber Angriffsflächen für Cyberkriminelle. Um die Daten sicher zu machen, hat Verschlüsselungsexperte IDENTOS "Secure Messaging" entwickelt, einen Kommunikationskanal, bei dem alle Daten Ende-zu-Ende verschlüsselt werden und zwar immer mit einem separaten Schlüssel. In Kanada wird das System, das sich unabhängig von der Software nutzen, aber eben auch auf das Kliniksystem aufsetzen lässt und dann mit dem Dienstplan kommunizieren kann, bereits von Ärzten genutzt. In Ontario sogar, um Rechnungen schneller und gesichert zum Gesundheitsministerium zu schicken. Um die App, die es für den Messaging-Dienst braucht, nutzen zu können, müssen sich die Ärzte zusätzlich mit einer PIN verifizieren. Verloren gegangene oder sabotierte Geräte lassen sich zudem zentral sperren. RebekkaHöhl

\section{Indamed will Videodienst in Software integrieren}

Der Praxis-EDV-Anbieter Indamed plant eine Videosprechstundenfunktion direkt aus seiner Arztsoftware Medical Office heraus. Dafür hat er sich als Partner TerMed mit ins Boot geholt. Sobald der Patient das virtuelle Wartezimmer betreten habe, werde dies in der Medical OfficeTagesliste angezeigt. Aus der Liste heraus könne der Arzt den Patienten dann in das "virtuelle Behandlungszimmer" ziehen und den Videokontakt beginnen. Wann genau die neue Funktion umgesetzt ist, lässt das Unternehmen allerdings noch offen. „Die Arbeiten laufen auf Hochtouren“, heißt es. Rebekka Höhl

\title{
Start-up setzt auf flexible Videosprechstunde
}

\author{
Ein gängiges Smartphone - mehr benötigen Ärzte und Patienten nicht, um \\ den Videosprechstundendienst des Start-ups Minxl nutzen zu können. Dabei \\ können Ärzte ihren Bericht einfach via App diktieren.
}

S eit die EBM-Ziffern für die Videosprechstunde stehen, ist der Patientenkontakt via Videoverbindung ein heiß diskutiertes Thema, denn die Auflagen, die KBV und GKV-Spitzenverband an die Technik stellen, sind nicht ohne. Unter anderem müssen Arzt und Patient eindeutig identifizierbar sein. Dennoch ist sich Jennifer Kelly, Gründerin und Geschäftsführerin des Videosprechstundendienstes Minxli sicher, dass ihr Dienst die Anforderungen erfüllt.

Das System ist für Ärzte tatsächlich flexibel einsetzbar. Alles, was sie und ihre Patienten benötigen, ist ein Smartphone mit dem Betriebssystem Android oder mit Apples OS X. Empfohlen wird von Minxli zwar eine WLAN-Verbindung, die Videoapplikation läuft aber auch über das mobile Internet. Der Arzt gibt auf Minxli innerhalb des Terminkalenders Zeitfenster für die Videosprechstunde ein. Der Patient kann dann Termine buchen und füllt vorab eine Patienteninformation mit allgemeinen $\mathrm{Da}$ ten - also Geburtsdatum, Geschlecht sowie medizinische Informationen $\mathrm{zu} \mathrm{Al}$ lergien und aktueller Medikation - aus, ähnlich dem Anamnesebogen in der Praxis. Damit kann der Patient eindeutig zugeordnet werden. Anrufen kann

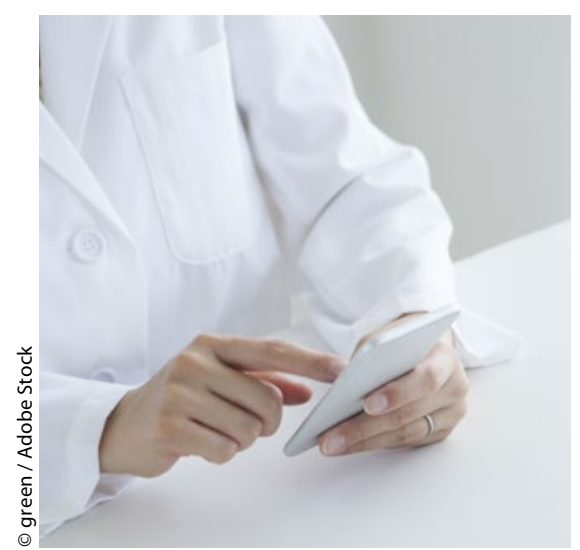

Videosprechstunde mit dem Arzt via Smartphone. jedoch nur der Arzt. Dadurch soll die Privatsphäre des Arztes geschützt werden. Außerdem zählt auch dies zu den Auflagen von KBV und Krankenkassen.

Spannend ist, dass der Arzt während des Videokontaktes zwischen reiner Chatfunktion und tatsächlichem Videotelefonat wechseln kann. Sowohl Patient als auch Arzt können Fotos und andere Dokumente mittels App austauschen. Mit der Smartphone-App kann der Patient dabei zum Beispiel Wundbilder sofort erfassen. Den Bericht zum Termin tippt der Arzt über das Smartphone ein oder er nutzt die Diktierfunktion. „Wir arbeiten gerade daran, unser System direkt mit Arztsoftwaresystemen zu verbinden", so Kelly. Noch können die Arztberichte nur via PDF gespeichert und dann beispielsweise per E-Mail in die Praxis-EDV übertragen werden.

Finanziert wird der Videodienst vom Arzt, dieser zahlt laut Kelly $1,99 €$ pro $\mathrm{Pa}$ tientenkontakt. Da die Ärzte auch ihr Profil im System hochladen und so für neue Patienten sichtbar sein können, arbeitet Minxli eng mit den Ärztekammern zusammen. Es werde für jeden Arzt geprüft, ob er auch tatsächlich eine gültige Approbation hat.

Die Daten werden verschlüsselt nach geltendem Sicherheitsstandard übertragen. Dabei stehen die Übermittlungsserver in Deutschland. Derzeit wird der Dienst vor allem von Ärzten genutzt, die Privatpatienten aus dem Ausland betreuen oder aber von Kliniken, die im Medizintourismus aktiv sind. Die Patienten stammten etwa aus Russland, Brasilien oder Saudi Arabien. Außerdem ermöglicht der Dienst Ärzten den Austausch mit Fachkollegen über einen gesonderten Expertenchat. Weil es bislang kaum Daten zur Effektivität von Videosprechstunden gibt, will das Start-up gemeinsam mit der Universitätsklinik Heidelberg eine Studie aufsetzen. Geplant ist, den Videokontakt bei der Betreuung von Krebspatienten zu evaluieren.

RebekkaHöhl 